\title{
The Minimum Barrier Distance - Stability to Seed Point Position
}

\author{
Robin Strand ${ }^{1}$, Filip Malmberg ${ }^{1}$, Punam K. Saha ${ }^{2}$, and Elisabeth Linnér ${ }^{1}$ \\ ${ }^{1}$ Centre for Image Analysis, Division of Visual Information and Interaction, \\ Uppsala University, Sweden \\ 2 Dept. of Electrical and Computer Engineering and Dept. of Radiology, University of Iowa, \\ Iowa City, IA 52242, United States
}

\begin{abstract}
Distance and path-cost functions have been used for image segmentation at various forms, e.g., region growing or live-wire boundary tracing using interactive user input. Different approaches are associated with different fundamental advantages as well as difficulties. In this paper, we investigate the stability of segmentation with respect to perturbations in seed point position for a recently introduced pseudo-distance method referred to as the minimum barrier distance. Conditions are sought for which segmentation results are invariant with respect to the position of seed points and a proof of their correctness is presented. A notion of $\delta$-interface is introduced defining the object-background interface at various gradations and its relation to stability of segmentation is examined. Finally, experimental results are presented examining different aspects of stability of segmentation results to seed point position.
\end{abstract}

\section{Introduction}

Distance transforms and functions are widely used in image processing [1-8]. Intensityweighted distance transforms take the pixel intensity values into consideration [6, 9-11]. This way, the homogeneity of intensity values in regions are quantified. This property makes intensity-weighted distances well-suited for image segmentation, where the goal is to group pixels in homogenous regions.

Here, image segmentation by intensity-weighted distances is achieved by assigning to each pixel the distance to, and the label of, the closest labeled seed point. The seed point can be given by a user or utilizing some domain knowledge. Stability to seed point position is a very important aspect of these segmentation methods; small perturbations in the seed point position should ideally not lead to significantly changed segmentation result. Different aspects on stability to seed point position for intensity-weighted distances have been examined [1, 12, 13].

Many distance- or cost functions in image segmentation by region growing have a locality property, which makes it possible to efficiently compute distance (or cost) defined as optimal paths by propagating values from adjacent points. The locality property is in one sense a deficiency, since global properties can not easily be included in the propagation. On the other hand, the locality property is essential for efficient computation, typically by wave-front propagation starting from points with zero cost or distance. 
A well-known and often used method, watershed, can intuitively be described as a flooding process of a topographic representation of gray scale images, where low intensities correspond to low altitude in the topographic representation. A recently developed method, the minimum barrier distance, is given by the minimum barrier that has to be passed to go from one point to another in the topographic representation of image data. The minimum barrier distance is a pseudo-metric, meaning that the properties identity, symmetry and triangle inequality, but not positivity, are obeyed [1]. We have showed that the minimum barrier distance has many properties that make it beneficial for image segmentation, e.g., stability to seed point position (using simple Dice's coefficient on segmentation results), noise, smoothing etc. [1, 13].

However the minimum barrier path cost function is not local in the above sense, and therefore standard wave-front propagation algorithms are not sufficient for computing the exact minimum barrier distance map [1, 13]. We have developed efficient algorithms for computing the minimum barrier distance [13] and approximations thereof [1]. A vectorial minimum barrier distance that takes multi-band image data, e.g., color or other multispectral images, as input has also been developed [14].

In this paper, we examine the stability to seed point position for the minimum barrier distance by introducing conditions under which the distance between points $p$ and $q$ equals the distance between $p$ and another point $q^{\prime}$. Also, we introduce the $\delta$-interface which essentially is a region of uncertainty of border position in a segmentation result.

\section{The Minimum Barrier Distance}

We will consider $D=\left\{p=\left(x_{1}, x_{2}, \ldots, x_{n}\right) \in \mathbb{Z}^{n}: L_{i} \leq x_{i} \leq U_{i}\right\}$ as the image domain. The intensity at a point $p$ is denoted by $f(p)$. A path, $\pi=\left\langle p_{0}, p_{1}, \ldots, p_{n}\right\rangle$, is a sequence of points $p_{0}, p_{1}, \ldots, p_{n}$, where each pair of consecutive points are adjacent given some adjacency. The maximum and minimum values along a path $\pi=$ $\left\langle p_{0}, p_{1}, \ldots, p_{n}\right\rangle$ are

$$
\max (\pi)=\max _{0 \leq i \leq n} f\left(p_{i}\right) \text { and } \min (\pi)=\min _{0 \leq i \leq n} f\left(p_{i}\right),
$$

respectively. The minimum barrier along a path $\pi$ is defined as

$$
\Phi(\pi)=\max (\pi)-\min (\pi) .
$$

The minimum barrier distance between two points $p$ and $q$ is defined as

$$
\Phi(p, q)=\min _{\pi \in \Pi} \Phi(\pi),
$$

where $\Pi$ is the set of all paths between $p$ and $q$. A path in $\Pi$ that attains the minimum barrier distance is called an MBD-optimal path. The minimax and maximin distances between points are defined as

$$
\Phi_{\max }(p, q)=\min _{\pi \in \Pi} \max (\pi) \text { and } \Phi_{\min }(p, q)=\max _{\pi \in \Pi} \min (\pi),
$$

respectively. The concatenation of two paths $\pi$ and $\tau$ is denoted $\pi \cdot \tau$.

In [1], we gave an approximation of the minimum barrier distance, namely $\Phi(p, q) \approx$ $\Phi_{\max }(p, q)-\Phi_{\min }(p, q)$. We showed that $\Phi_{\max }-\Phi_{\min }$ equals $\Phi$ in the continuous case and converges to $\Phi$ as the sampling density increases in the discrete case [1]. 


\section{Distance Transform Algorithms and Segmentation}

As previously mentioned, the key to seeded segmentation by intensity weighted distance transforms is the distance transform, where each pixel is assigned the distance to (and label of) the closest seed point. We have developed different algorithms that compute approximations of the minimum barrier distance-transform in $O(n \log n)$ time, where $n$ is the number of pixels [1, 13]. In this manuscript, we will use our recently developed efficient algorithm for computing the exact minimum barrier distance [13]. The worst case time complexity is $O(m n \log n)$ (or $O(m(n+m)$ ), depending on the data structure), where $m$ is the range of the weight function (here the number of intensitites that can be attained).

\section{Stability of the Minimum Barrier Distance with Respect to Seed Point Position}

In this section, we introduce this paper's theoretical results on conditions for which the minimum barrier distance between $p$ and $q$ equals the distance between $p$ and $q^{\prime}$ and the $\delta$-interface. The latter will also be used in the experiments in Section 4

\subsection{Invariance under Seed Point Position}

In interactive segmentation, there is an uncertainty in the exact positions of seed points due to, for example, inter- and intra-user variability. Given a point $p$ (for example in the object) and a user added seed point $q$ (for example in the background). The used distance function has a high invariance under seed point position if there is a large set of points that can be used instead of $q$ without altering the distance to the point $p$. Following this idea, conditions for which the minimum barrier distance value between a point $p$ and seed point $q$ equals the minimum barrier distance value between $p$ and another seed point $q^{\prime}$ are given in the following theorem.

Theorem 1. Let $q$ and $q^{\prime}$ be points, and let $I=\left[I_{\min }, I_{\max }\right]$ be an interval such that any MBD-optimal paths $\pi$ between $q$ and $q^{\prime}$ are such that $\max (\pi) \leq I_{\max }$ and $\min (\pi) \geq$ $I_{\min }$. Let $p$ be a point such that

- $\Phi_{\max }(q, p)>I_{\max } \cdot(\dagger)$

- $\Phi_{\min }(q, p)<I_{\min } .(\ddagger)$

Then $\Phi\left(q^{\prime}, p\right)=\Phi(q, p)$.

Proof. Let $\pi$ be an MBD-optimal path between $p$ and $q^{\prime}$, and let $\pi^{\prime}$ be an MBD-optimal path between $q$ and $q^{\prime}$. Then $\Phi\left(\pi \cdot \pi^{\prime}\right)=\max \left(\max (\pi), \max \left(\pi^{\prime}\right)\right)-\min (\min (\pi)$, $\left.\min \left(\pi^{\prime}\right)\right)=\max \left(\pi^{\prime}\right)-\min \left(\pi^{\prime}\right)=\Phi\left(\pi^{\prime}\right)$, and so $\Phi\left(q^{\prime}, p\right) \leq \Phi(q, p)$.

Next, assume $(*)$ that there exists a path $\pi^{\prime \prime}$ between $q^{\prime}$ and $p$ such that $\Phi\left(\pi^{\prime \prime}\right)<$ $\Phi(q, p)$. Then the following properties hold:

- $\max \left(\pi^{\prime \prime}\right) \geq \Phi_{\max }(q, p)$. (Otherwise $\max \left(\pi \cdot \pi^{\prime \prime}\right)<\Phi_{\max }(q, p)$, contradicting $(\dagger)$.)

- $\min \left(\pi^{\prime \prime}\right) \leq \Phi_{\min }(q, p)$. (Otherwise $\min \left(\pi \cdot \pi^{\prime \prime}\right)<\Phi_{\min }(q, p)$, contradicting (†).) 
From these properties, it follows that $\Phi\left(\pi \cdot \pi^{\prime \prime}\right)=\Phi\left(\pi^{\prime \prime}\right)$. But $\pi \cdot \pi^{\prime \prime}$ is a path between $q$ and $p$ and therefore $\Phi\left(\pi^{\prime \prime}\right) \nless \Phi(q, p)$, contradicting (*).

Note that the strong conditions in Theorem 1 are not often satisfied for real images. The theorem and conditions are presented here mainly to give a deeper understanding of the minimum barrier distance.

\subsection{The $\delta$-Interface}

In region growing segmentation among multiple objects [15], different objects are defined with respect to their seed points where a given point is assigned to an object whose seeds are closest to that point under a cost- or distance-function. Here, we formulate a notion of $\delta$-interface that defines the region of uncertainty at the interface between two or more objects. Ideally, small perturbation in seed point positions will only effect the segmentation region within the uncertain region and, therefore, segmented core objects regions will be unaltered under such small perturbation in seed positions. The $\delta$-interface will be used as a tool to quantify this stability.

The $\delta$-interface is a region with 'thickness' $\delta$ where two regions meet. A $\delta$-interface with $\delta=0$ is the set of points located at the exact same distance to two seed points. Given a value of $\delta \geq 0$, the $\delta$-interface between points $p$ and $q$ is the set $I(p, q, \delta)=$ $\{r:|\Phi(p, r)-\Phi(q, r)| \leq \delta\}$.

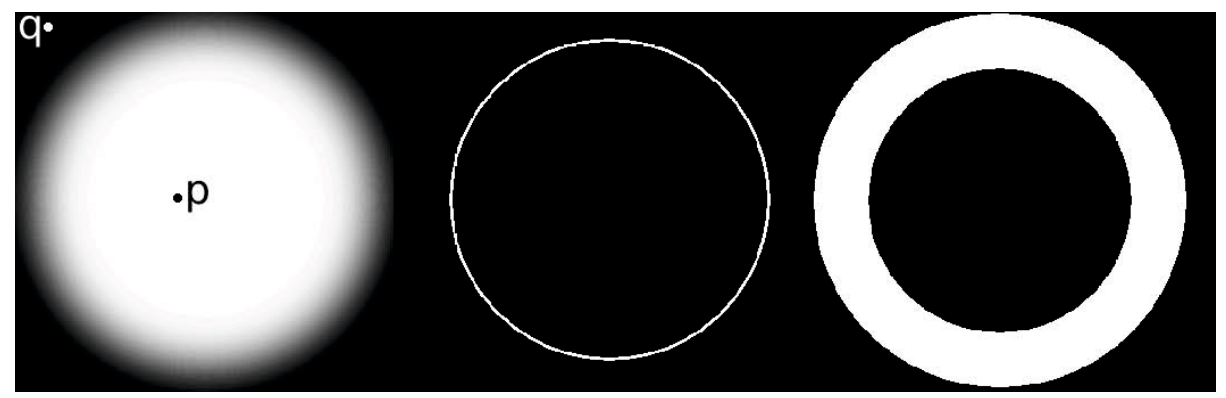

Fig. 1. Illustration of $\delta$-interfaces. Left: example image and points $p$ and $q$. Middle: $I(p, q, \delta)$ (shown in white), with small $\delta$. Right: $I(p, q, \delta)$, with large $\delta$.

The following Theorem gives a correspondence between the delta-interface between $p, q$ and $p, q^{\prime}$.

Theorem 2. $I(p, q, \delta) \subset I\left(p, q^{\prime}, \delta+\Phi\left(q, q^{\prime}\right)\right)$

Proof. Let $r \in I(p, q, \delta)$. We want to show that $r \in I\left(p, q^{\prime}, \delta+\Phi\left(q, q^{\prime}\right)\right)$, i.e. that

$$
\left|\Phi\left(q^{\prime}, r\right)-\Phi(p, r)\right| \leq \delta+\Phi\left(q, q^{\prime}\right) .
$$

(i) First of all, by the triangular inequality, $\Phi\left(q^{\prime}, r\right)-\Phi(p, r) \leq \Phi(q, r)+\Phi\left(q, q^{\prime}\right)-$ $\Phi(p, r) \leq \delta+\Phi\left(q, q^{\prime}\right)$. 
(ii) Secondly, by the triangle inequality, $|\Phi(r, p)-\Phi(q, p)| \leq \Phi(q, r)$ for any given points $p, q, r$. Therefore, $\Phi\left(q^{\prime}, r\right)-\Phi(p, r) \geq \Phi(q, r)-\Phi\left(q, q^{\prime}\right)-\Phi(p, r) \geq-\delta-$ $\Phi\left(q, q^{\prime}\right)$. By combining (i) and (ii), we get exactly (1).

The following Corollary gives a set containing borders between objects defined by seed points $p, q$ and $p, q^{\prime}$, respectively. The smaller the set, the more robust the minimum barrier distance is to seed point positioning.

Corollary 1. $I(p, q, \delta), I\left(p, q^{\prime}, \delta\right) \subset\left(I\left(p, q^{\prime}, \delta+\Phi\left(q, q^{\prime}\right)\right) \cap I\left(p, q, \delta+\Phi\left(q, q^{\prime}\right)\right)\right)$

The sets $I\left(p, q^{\prime}, \Phi\left(q, q^{\prime}\right)\right)$ and $I\left(p, q, \Phi\left(q, q^{\prime}\right)\right)$, i.e. when $\delta=0$, are illustrated in Figure 2

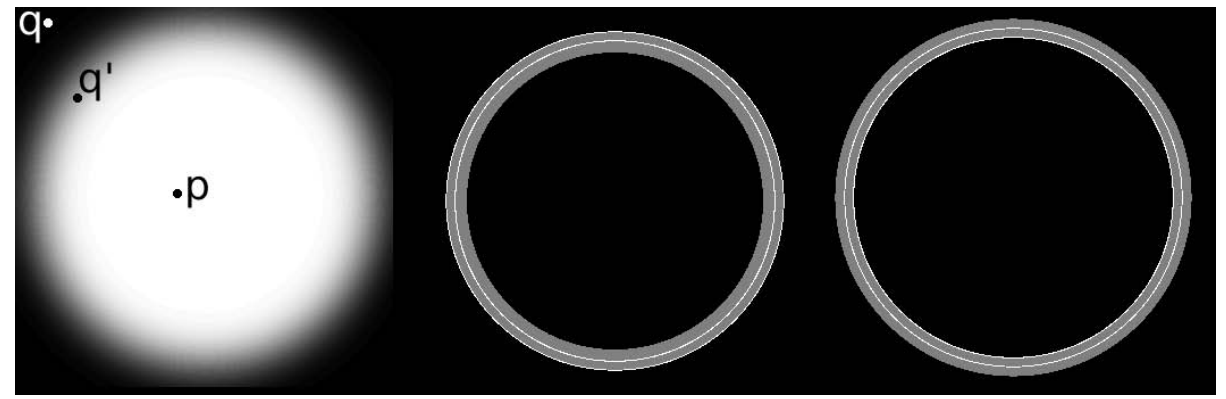

Fig. 2. Illustration of Corollary 1 and the experiment in Section 4.1 Left: A gray-level image with three seed-points, $p, q$, and $q^{\prime}$. Middle: The set $I\left(p, q^{\prime}, \Phi\left(q, q^{\prime}\right)\right)$ is shown in gray. The sets $I(p, q, 0)$ and $I\left(p, q^{\prime}, 0\right)$ are shown in white. Right: The set $I\left(p, q, \Phi\left(q, q^{\prime}\right)\right)$ is shown in gray. The sets $I(p, q, 0)$ and $I\left(p, q^{\prime}, 0\right)$ are shown in white. $E\left(p, q, q^{\prime}, 0\right)$ is the intersection of $I\left(p, q^{\prime}, \Phi\left(q, q^{\prime}\right)\right)$ and $I\left(p, q, \Phi\left(q, q^{\prime}\right)\right)$.

\section{Experiments and Results}

Seventeen images from the grabcut dataset [16] are used for the experiments, see Figure 3. The images come with a reference segmentation, which we eroded/dilated to get object and background regions as shown for an example image in Figure 4 The images, converted to gray scale by using the mean of the three color band values, are used. The intensity range of the images is $[0,255]$.

\subsection{Stability to Seed Point Position}

Seed points added by a user with low accuracy or precision will differ in spatial distance. Ideally, a small perturbation in the seed point position gives a small difference in the segmentation result. In this section, we will evaluate the effect of a small change in position of the seed point $q$. This will be done by comparing the $\delta$-interface of $p$ and $q$ with that of $p$ and $q^{\prime}$ (such that $q$ and $q^{\prime}$ are at a fixed spatial distance). We will see how 


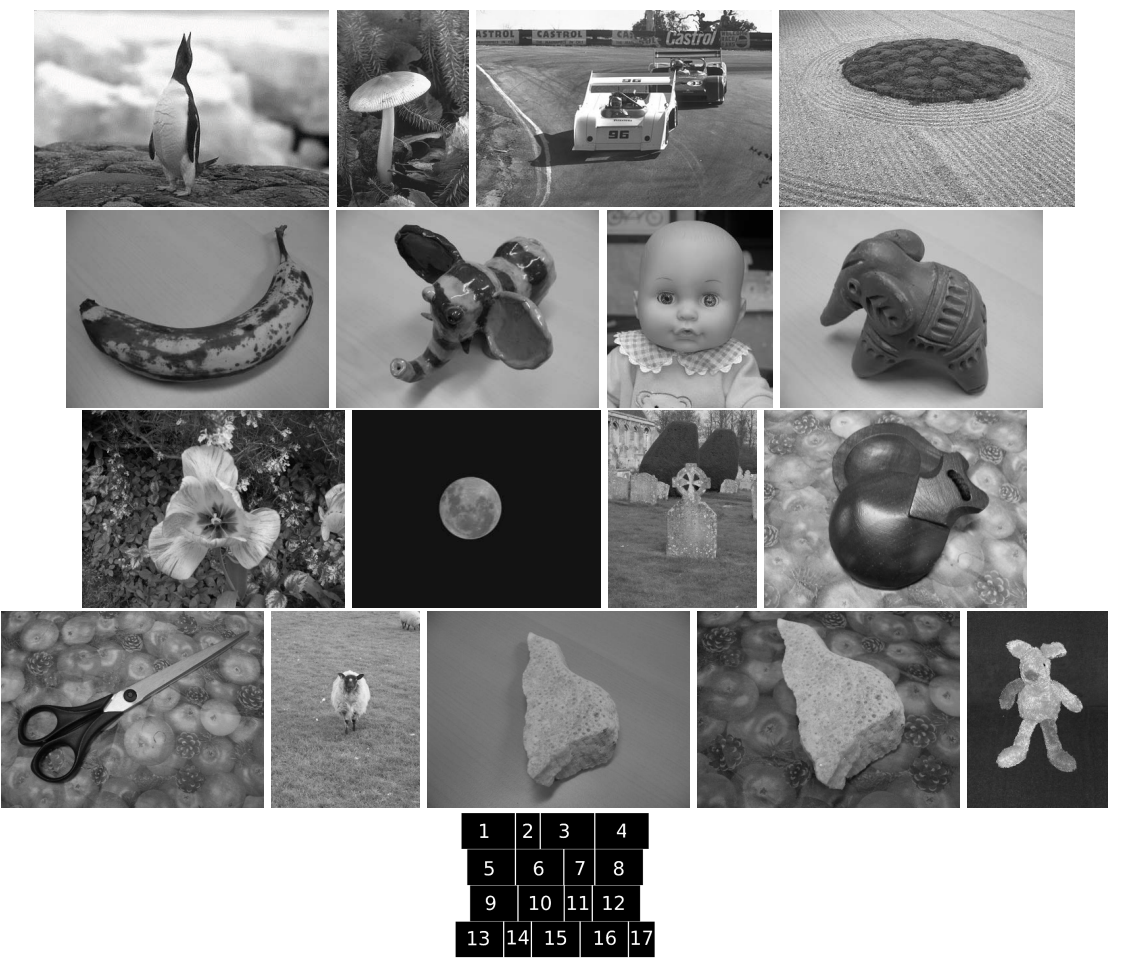

Fig. 3. Images from the grabcut database used for the experiments. Bottom: image numbering.
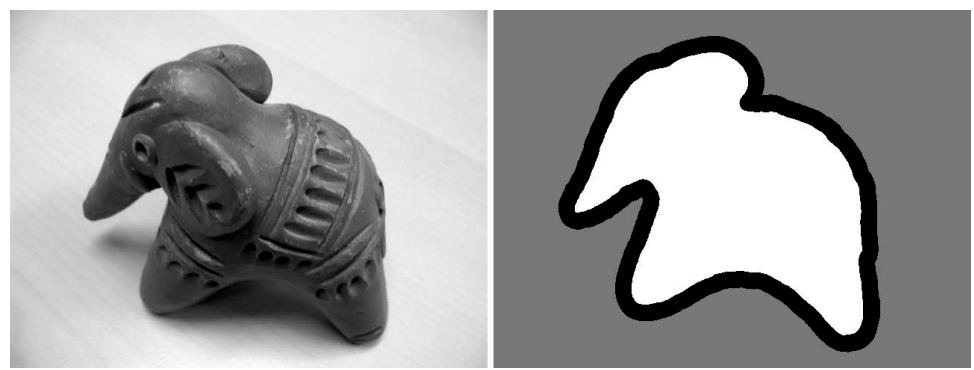

Fig. 4. Image 8 from the grabcut database used for the experiments. Left: original image. Right: Object region (white) and background region (gray). 
the spatial distance between seed points $q$ and $q^{\prime}$ affect the $\delta$-interface. The set metric we will use in the evaluation is

$$
E\left(p, q, q^{\prime}, \delta\right)=\left|I\left(p, q^{\prime}, \delta+\Phi\left(q, q^{\prime}\right)\right) \cap I\left(p, q, \delta+\Phi\left(q, q^{\prime}\right)\right)\right|
$$

with $\delta=0$. The set $I(p, q, 0)$ corresponds exactly to the set of pixels with equal distance between $p$ and $q$. In other words, intuitively, this is the border between the regions that correspond to $p$ and $q$, respectively in a segmentation. By comparing this set and the set obtained by $I\left(p, q^{\prime}, 0\right)$, where $q^{\prime}$ is a point close to $q$, the difference between a
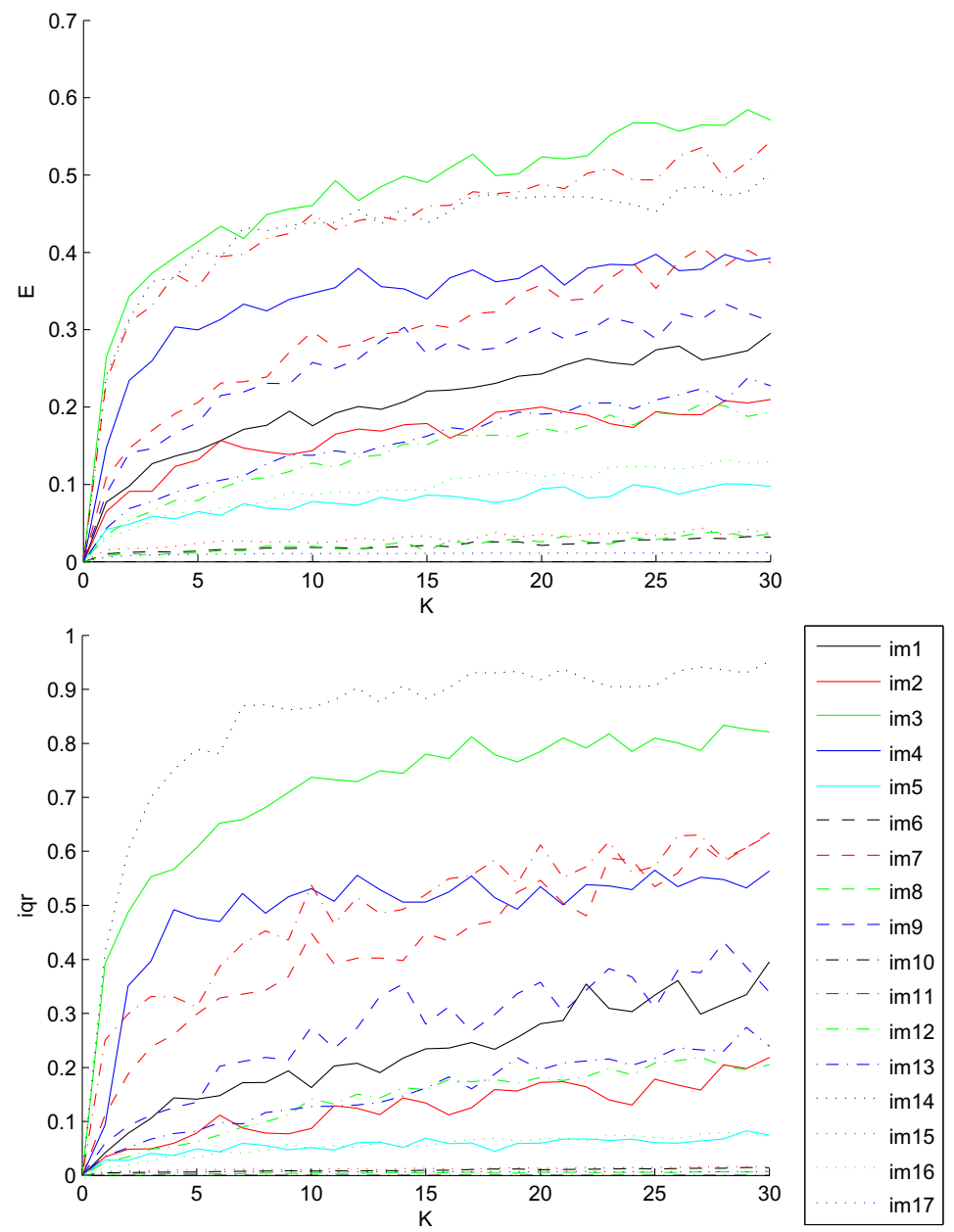

Fig. 5. Cardinality of the set $E\left(p, q, q^{\prime}, 0\right)$, for random points $p$ (in the object), $q$ (in the background), and $q^{\prime}$ (in the background such that $\left\|q-q^{\prime}\right\|=K$ ) as a function of $K$. The mean values (top) and the interquartile range (iqr, the difference of the 75:th and 25:th percentiles, bottom) of 1000 executions for each image and value of $K$ are plotted. The values are normalized with the size (number of pixels) of the images. 
segmentation result obtained by seed points $p$ and $q$ compared to $p$ and $q^{\prime}$ can be quantified. The quantification we will use is $E\left(p, q, q^{\prime}, 0\right)$, for random points $p$ (in the object), $q$ (in the background), and $q^{\prime}$ (in the background and such that $\left\|q-q^{\prime}\right\|$ approximately equals a constant $K$ ). See the illustration in Figure 2

Note that the quantification $E$ used in the experiment only measures the difference between the interface $I(p, q, 0)$ and the interface $I\left(p, q^{\prime}, 0\right)$. Clear distinction by the distance function between object and background gives a small interface and the lower $E$ is, the smaller the interface is. See Figure 1 and Figure 2 for illustrations.
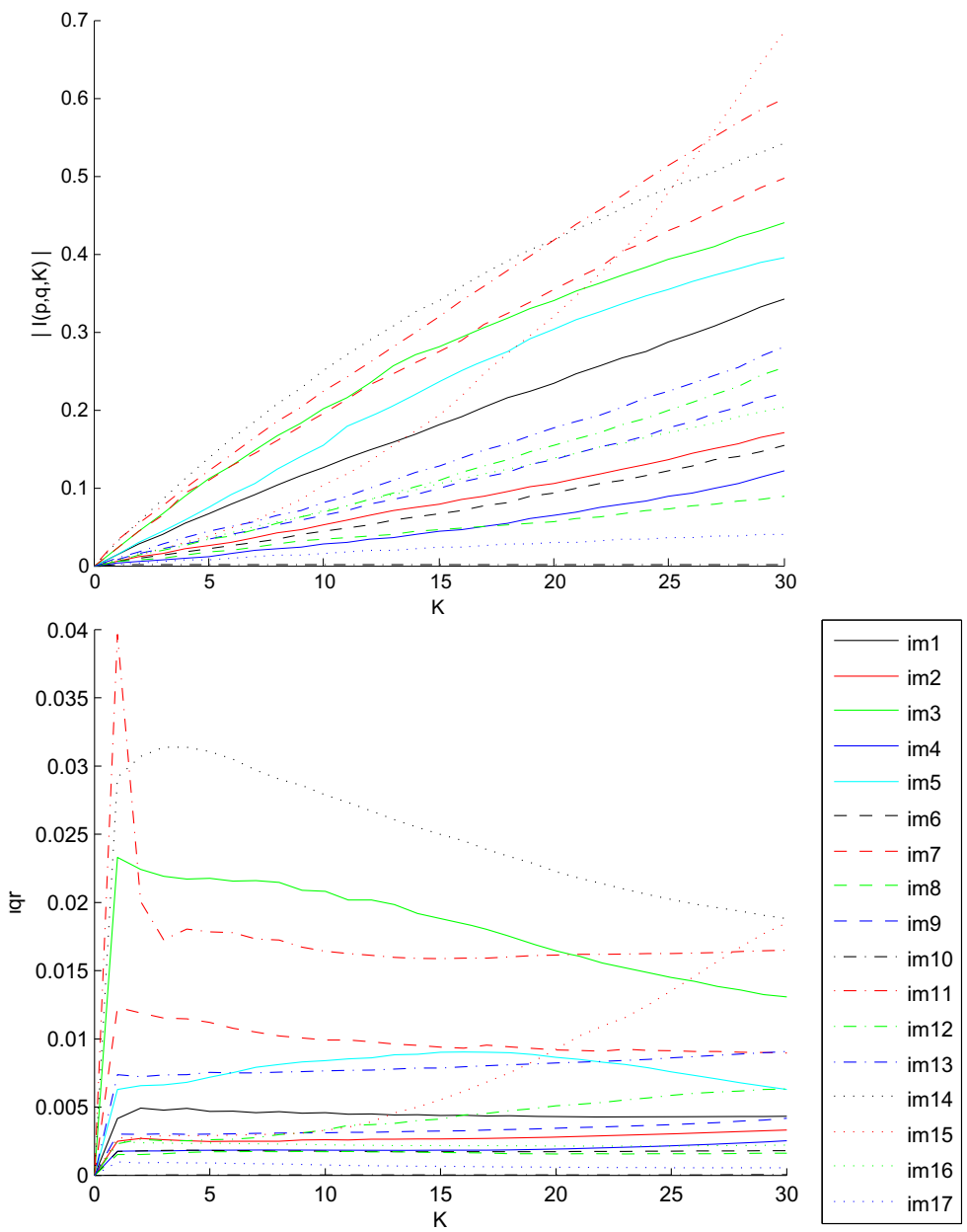

Fig. 6. Cardinality of the set $I(p, q, K)$ as a function of $K$. For each of the 17 images in Figure 3 $I(p, q, K)$, where $q$ is a random object seed point and $p$ is a random background seed point, is generated for $K=1 \ldots 30$. The mean values (top) and the interquartile range (iqr, bottom) of 1000 executions for each image and value of $K$ are plotted. The values are normalized with the size (number of pixels) of the images. 
Since the cardinality of the set $\left\{q^{\prime} \in \mathbb{Z}^{2}:\left\|q-q^{\prime}\right\|=K\right\}$ usually is very small in the digital space, we can not use points $q$ and $q^{\prime}$ at exactly a Euclidean distance $K$. Instead, points $q^{\prime}$ are derived as follows: a random angle $\theta \in[0,2 \pi$ [ is extracted and the point $q^{\prime}=q+(\lfloor K \cos \theta\rceil,\lfloor K \sin \theta\rceil)$ is used. Here, $\lfloor\cdot\rceil$ is the rounding off function which gives the nearest integer.

A plot showing the mean overlap region from 1000 iterations per value of $K$ for the 17 images in Figure 3 is shown in Figure 5.

\subsection{Uncertainty of Border Position}

The idea behind this experiment is to see how the cardinality of the $\delta$-interface increases with increasing values of $\delta$. By iteratively and randomly selecting an object seed point $p$ and a background seed point $q$ and computing the cardinality of the set $I(p, q, K)$ for increasing values of $K$, quantitative measures on the region where the uncertainty of the border position is high can be obtained. A plot on the mean cardinality of the uncertainty region from 1000 iterations per value of $K$ for the 17 images in Figure 3 is shown in Figure 6.

\section{Conclusions and Future Work}

In Theorem 1, we gave conditions that guarantee that the minimum barrier distance between $p$ and $q$ equals the distance between $p$ and a third point $r$. The conditions are correct by the proof. However, the practical implications of Theorem 1 are limited since the conditions are not often satisfied in real world images. Therefore, no evaluation of Theorem 1 is presented here.

In the first experiment, we evaluated how small perturbations in the seed point position changed the segmentation result by a measure on the difference between object region border when $p$ and $q$ are used as seed points, compared to when $p$ and $q^{\prime}$, such that $\left\|q-q^{\prime}\right\| \approx K$, are used. From the plots in Figure 5 we can see that there is a big difference between the images with lowest error (image 10, 17, 6, 8, 15, 5 in Figure 3 ) and the images with highest error (image 3, 11, 14, 4, 7, 9). The low-error images all have a distinct border between object and background, whereas this border is not as distinct in the images with high error. Also, images with an apparent texture all give high error. This is expected since only barriers based on intensity are taken into account in the minimum barrier distance given here. The vectorial minimum barrier distance, presented in [14], with appropriate point-wise texture features is expected to handle these situations better.

Statistical dispersions were computed by the interquartile range (the difference of the 75:th and 25:th percentiles) for the experiments, see Figure 5 and Figure 6 For experiment 1 , the general trend was that the higher mean value, the higher interquartile distance. The interquartile distance values were roughly in the same range as the mean values. For experiment 2, the dispersion measures were lower, suggesting that these results are more reliable.

In the second experiment, the region with pixels such that the distances to the two seed points $p$ and $q$ are similar $(K)$ is quantified. In most cases, a linear relationship 
between $K$ and the cardinality of the $\delta$-interface was observed. This relationship seems to not hold for image 15 , probably due to the weak gradient in the border between object and background. Judging from the images with the lowest error (image 10, 17, 8, 4, 6, 2 ) and the images with highest error at $K=30$ (image $15,11,14,7,3,5$ ), we conclude that this error measure seems to be less sensitive to texture.

In our future work, we plan to combine the minimum barrier distance with a spatial distance term to avoid problems with object 'leakage' due to the fact that after passing a large 'barrier', distance values are constant. Experiment 1 in this paper evaluates how small perturbations in the seed point positions change the segmentation result. As a result of the insightful reviews of this paper, we plan to define an uncertainty model from a probability displacement distribution and then to compute statistics while sampling $q^{\prime}$ in this distribution. This approach will more realistically model the distribution of seed points added by a large number of users. We also plan to do extensive evaluation of stability of several intensity-weighted distance functions, and methods that can be expressed as intensity-weighted distance- or cost-functions such as [17], with respect to seed point position, blur, inhomogeneity, noise, etc.

\section{References}

1. Strand, R., Ciesielski, K.C., Malmberg, F., Saha, P.K.: The minimum barrier distance. Computer Vision and Image Understanding 117(4), 429-437 (2013), special issue on Discrete Geometry for Computer Imagery

2. Rosenfeld, A., Pfaltz, J.L.: Distance functions on digital pictures. Pattern Recognition 1, 33-61 (1968)

3. Borgefors, G.: Distance transformations in arbitrary dimensions. Computer Vision, Graphics, and Image Processing 27, 321-345 (1984)

4. Borgefors, G.: On digital distance transforms in three dimensions. Computer Vision and Image Understanding 64(3), 368-376 (1996)

5. Danielsson, P.-E.: Euclidean distance mapping. Computer Graphics and Image Processing 14, 227-248 (1980)

6. Saha, P.K., Wehrli, F.W., Gomberg, B.R.: Fuzzy distance transform: theory, algorithms, and applications. Computer Vision and Image Understanding 86, 171-190 (2002)

7. Strand, R.: Distance functions and image processing on point-lattices: with focus on the 3D face- and body-centered cubic grids, Ph.D. thesis, Uppsala University, Sweden (2008), http://urn.kb.se/resolve?urn=urn:nbn:se:uu:diva-9312

8. Strand, R., Nagy, B., Borgefors, G.: Digital distance functions on three-dimensional grids. Theoretical Computer Science 412(15), 1350-1363 (2011)

9. Sethian, J.A.: Level Set Methods and Fast Marching Methods. Cambridge University Press (1999)

10. Fouard, C., Gedda, M.: An objective comparison between gray weighted distance transforms and weighted distance transforms on curved spaces. In: Kuba, A., Nyúl, L.G., Palágyi, K. (eds.) DGCI 2006. LNCS, vol. 4245, pp. 259-270. Springer, Heidelberg (2006)

11. Falcão, A.X., Stolfi, J., Lotufo, R.A.: The image foresting transform: Theory, algorithms, and applications. IEEE Transactions on Pattern Analysis and Machine Intelligence 26(1), 19-29 (2004)

12. Audigier, R., Lotufo, R.A.: Seed-relative robustness of watershed and fuzzy connectedness approaches. In: Brazilian Symposium on Computer Graphics and Image Processing. IEEE (2007) 
13. Ciesielski, K.C., Strand, R., Malmberg, F., Saha, P.K.: Efficient algorithm for finding the exact minimum barrier distance. Computer Vision and Image Understanding 123, 53-64 (2014)

14. Kårsnäs, A., Strand, R., Saha, P.K.: The vectorial minimum barrier distance. In: 2012 21st International Conference on Pattern Recognition (ICPR), pp. 792-795 (2012)

15. Ciesielski, K.C., Udupa, J.K., Saha, P.K., Zhuge, Y.: Iterative relative fuzzy connectedness for multiple objects with multiple seeds. Computer Vision and Image Understanding 107(3), 160-182 (2007)

16. Rother, C., Kolmogorov, V., Blake, A.: GrabCut: Interactive Foreground Extraction using Iterated Graph Cuts. ACM Transactions on Graphics, SIGGRAPH 2004 (2004)

17. Bertrand, G.: On topological watersheds. Journal of Mathematical Image and Vision 22(2-3), 217-230 (2005) 